\title{
Article \\ A Prospective, Real-World, Multinational Study of Naloxegol for Patients with Cancer Pain Diagnosed with Opioid-Induced Constipation-The NACASY Study
}

\author{
Andrew Davies ${ }^{1, * \mathbb{D}}$, Saverio Cinieri ${ }^{2}$, Denis Dupoiron ${ }^{3} \mathbb{D}$, Sofia España Fernandez ${ }^{4} \mathbb{D}$, Johan Leclerc ${ }^{5}$, \\ Vincenzo Montesarchio ${ }^{6}$, Kyriaki Mystakidou ${ }^{7}$, Judith Serna ${ }^{8}$, Jan Tack ${ }^{9}$ \\ and on behalf of the NACASY Study Group ${ }^{\dagger}$
}

check for updates

Citation: Davies, A.; Cinieri, S.; Dupoiron, D.; España Fernandez, S.; Leclerc, J.; Montesarchio, V.;

Mystakidou, K.; Serna, J.; Tack, J.; on behalf of the NACASY Study Group. A Prospective, Real-World,

Multinational Study of Naloxegol for Patients with Cancer Pain Diagnosed with Opioid-Induced

Constipation-The NACASY Study.

Cancers 2022, 14, 1128. https://

doi.org/10.3390/cancers14051128

Academic Editor: Sebastiano

Mercadante

Received: 30 January 2022

Accepted: 17 February 2022

Published: 23 February 2022

Publisher's Note: MDPI stays neutral with regard to jurisdictional claims in published maps and institutional affiliations.

Copyright: (C) 2022 by the authors. Licensee MDPI, Basel, Switzerland. This article is an open access article distributed under the terms and conditions of the Creative Commons Attribution (CC BY) license (https:// creativecommons.org/licenses/by/ $4.0 /)$.
1 Trinity College Dublin, University College Dublin, Our Lady's Hospice Dublin', D6W RY72 Dublin, Ireland 2 Medical Oncology, ASL Brindisi-P.O. “A. Perrino", 72100 Brindisi, Italy; saverio.cinieri@gmail.com

3 Département d'Anesthésie-Douleur, Institut de Cancerologie de l'Ouest-Site Paul Papin, 49055 Angers, France; denis.dupoiron@ico.unicancer.fr

4 Medical Oncology Department, Institut Català d'Oncologia-Badalona, 08916 Barcelona, Spain; sofia.ef@iconcologia.net

5 Doleur, CHU Amiens Picardie, 80054 Amiens, France; johan.leclerc@wanadoo.fr

6 UOC Ongologia, A.O.R.N. dei Colli-Monaldi-Coutgno-C.T.O. Hospitals, 80131 Napoli, Italy; vincenzo.montesarchio@ospedalideicolli.it

7 Palliative Care Unit "Jenny Karezi", School of Medicine, University of Athens, 11526 Athens, Greece; mistakidou@yahoo.com

8 Hospital Universitari Vall D’hebron, 08035 Barcelona, Spain; jserna@vhebron.net

9 University Hospital Gasthuisberg, 3000 Leuven, Belgium; jan.tack@kuleuven.be

* Correspondence: andavies@tcd.ie

+ Further membership of the NACASY Study Group is provided in the Acknowledgments.

Simple Summary: Prescription opioid pain medications help ease pain, but they also cause some unwanted side effects such as constipation. In this study we evaluated the safety and efficacy of naloxegol used to treat constipation that is caused by opioids. We found that naloxegol improved constipation and quality of life in patients with cancer-related pain and opioid-induced constipation.

Abstract: The Naloxegol Cancer Study (NACASY) was a multinational European study aimed to evaluate the 4-week safety and efficacy of naloxegol in a real-world setting in patients with cancer pain diagnosed with opioid-induced constipation. The primary safety endpoint was the incidence of adverse events leading to study discontinuation. We recruited 170 patients who received at least one dose of naloxegol (i.e., safety population). Out of 170 patients, 20 (11.8\%, 95\%CI 6.9-16.6) discontinued the study due to adverse events, and, of them, 12 (7.1\%, 95\%CI 3.2-10.9\%) were study discontinuations due to naloxegol-related adverse events. From 76 patients subjects who had completed both 4 weeks of treatment and 28 days of the diary, 55 patients (72.4\%, $95 \%$ CI $62.3-82.4 \%$ ) were regarded as responders (i.e., showed $\geq 3$ bowel-movements per week and an increase of $\geq 1$ bowel-movement over baseline) to naloxegol treatment. The Patient Assessment of ConstipationQuality of Life Questionnaire total score and all its subscales improved from baseline to 4 weeks of follow up. Our findings support and provide new evidence about the beneficial effect of naloxegol in terms of improvement of constipation and quality-of-life in patients with cancer-related pain and opioid-induced constipation and show a safety profile consistent with previous pivotal and real-world studies.

Keywords: naloxegol; PAMORA; MOVANTIK; opioid-induced constipation; cancer pain; real-world

\section{Introduction}

Cancer-related pain is one of the most frequent and bothersome symptoms affecting patients with cancer [1]. The frequency of cancer pain is related to the stage of disease. Pain 
prevalence increases from $33 \%$ in patients after curative treatment up to $64 \%$ in patients with metastatic, advanced, or terminal disease [2].

Opioids remain the cornerstone of analgesic treatment for severe cancer pain $[3,4]$. However, many opioid-associated adverse events may compromise their utilization [5]. Among these, opioid-induced constipation (OIC) is the most common and debilitating reported side-effect, with an overall prevalence ranging from $51 \%$ to $87 \%$ [6]. OIC negatively impacts patients' health-related quality of life (HRQoL), and impairs patients' ability to perform daily activities [7]. Hence, many patients decide to discontinue or limit their opioid therapy, resulting in insufficient pain control [8].

From a pathophysiological standpoint, OIC is predominantly the result of the agonistbinding and subsequent action of opioids on the peripheral network of $\mu$-opioid receptors in the gastrointestinal tract [7,9]; this leads to a constellation of effects, such as impaired esophageal motility, slowing of gastric emptying, and extended intestinal transit times, reduced intestinal secretions, and increased tone in intestinal sphincters [6]. Therefore, OIC treatment strategies should specifically address opioid binding to the GI peripheral $\mu$-opioid receptors [6].

Dietary modifications and lifestyle changes, followed by over-the-counter (OTC) stool softeners and laxatives, are the first-step management recommendations for OIC $[7,10]$. Nevertheless, the efficacy of OTC laxatives is limited, because they do not address the underlying pathophysiological cause of OIC $[9,11,12]$. Furthermore, in addition to lack of benefit $[7,8,10]$, several laxative associated side-effects, such as bloating, nausea, fecal incontinence, dehydration, and electrolyte imbalance, are frequently reported [13,14].

Peripherally acting $\mu$-opioid receptor antagonists (PAMORAs) constitute a novel class of drugs indicated for OIC that specifically bind and block the $\mu$-opioid receptors of the gastrointestinal tract, without crossing the blood-brain barrier and, hence, without interacting with the central nervous system (CNS) opioid receptors. Therefore, they have the ability to alleviate OIC without compromising the opioid's analgesic effects [11,12]. In patients who do not respond to standard laxatives, PAMORAs are a valid therapeutic option [15,16].

Naloxegol, a PEGylated derivate of naloxone, was the first orally administered PAMORA approved in 2014 for the treatment of OIC $[17,18]$. It is indicated for the treatment of OIC in noncancer and cancer adult patients with an inadequate response to laxatives; the recommended dosage is $25 \mathrm{mg}$ once daily and, if this is not tolerated, the dose could be reduced to $12.5 \mathrm{mg}$ once daily [18].

The clinical safety and efficacy of naloxegol was demonstrated in two identical phase III, 12-week, randomized, double-blind, placebo-controlled clinical trials, conducted in adult outpatients with noncancer pain and OIC (KODIAC-04 and KODIAC-05) [19]. Furthermore, the good safety and tolerability profile reported in these two studies was later corroborated in a 52-week trial in patients with noncancer pain and OIC, and in its further 12-week extension (KODIAC-08) [20,21].

Recently, two single-country real world studies demonstrating the efficacy and safety of naloxegol in cancer patients with OIC have been published [22,23]. However, data regarding the use of naloxegol in this group of patients in daily clinical practice across Europe do not exist.

We conducted the NACASY study, a multinational European study with the aim to evaluate the safety and efficacy of naloxegol in a real-world setting in patients with cancer pain diagnosed with OIC, during a 4-week follow-up period.

\section{Material and Methods}

\subsection{Study Design}

This was a four-week, single-arm, open-label, multinational, multicenter, prospective, real-world observational study in adult subjects with OIC, receiving naloxegol in routine clinical practice, as prescribed by their physicians according to the conditions established in its Summary of Product Characteristics (SmPC) and with the further recommendation to halt all currently used maintenance laxative therapy. 
A total of twenty-six European hospitals participated in the study. The study was conducted according to the requirements expressed in the Declaration of Helsinki [24], abiding to Good Epidemiological Practices [25] and current European regulations relating to the conduct of observational studies. The study was reviewed and approved by the Ethics Committees from the participating sites according to specific local regulations. Written informed consent was obtained from all subjects before enrollment in the study. ClinicalTrials.gov (accessed on 25 November 2021) Identifier: NCT03638440.

\subsection{Study Population}

Patients were eligible for the study if they met all the following inclusion criteria: adult patients ( $\geq 18$ years of age) with cancer pain, who had been receiving treatment with opioids for at least 4 weeks, were expected to remain on opioids for the entire duration of the study, and had been diagnosed with OIC (defined as $<3$ documented spontaneous bowel movements [SBMs] per week on average within the previous 2 weeks). In addition, patients must have reported $\geq 2$ of the following symptoms in at least $25 \%$ of the bowel movements (BMs) during that period: lumpy (Type 2) or hard (Type 1) stools, according to the Bristol Stool Scale (BSS); straining; sensation of incomplete BM; sensation of anorectal obstruction or blockage; a need for manual maneuvers to facilitate BMs; and, finally, loose stools rarely present without the use of laxatives. These criteria are consistent with the Rome IV diagnostic criteria for OIC [26,27].

Patients were excluded from the study if they were diagnosed with colorectal cancer.

\subsection{Study Outcomes and Assessments}

All the study data were obtained from routine clinical records, from information collected in the diaries provided to the enrolled subjects, or from the selected study questionnaires, and transcribed onto an anonymous case report form (CRF), which was divided into three follow-up sections: Visit 1 (baseline information); Visit 2 (Week 2); and Visit 3 (end of study: at Week 4 after naloxegol initiation).

The following variables were collected along the course of the study: (1) demographic data, cancer and pain clinical characteristics, and treatments; (2) OIC symptoms, laxative treatments, and BMs; (3) naloxegol treatment: initial dose and dose modifications throughout the study follow-up period; (4) all adverse events (AEs), whether related or not to naloxegol treatment, registered and coded according to MedDRA preferred terms (PTs), with their severity consequently graded using the National Cancer Institute Common Terminology Criteria for Adverse Events (CTCAE) v4.03; (5) laxative rescue medication; and 6) the following study questionnaires: (a) the Bowel Function Index (BFI), a physicianadministered questionnaire consisting of a three-item patient-assessment scale (ease of defecation, feeling of incomplete bowel evacuation, and personal judgement of constipation); (b) the BSS assessing the stool consistency at the time of every single BM, classifying them into 7 categories (from 1 indicating small, hard, lumpy stool, to 7 denoting watery stool); (c) straining perception assessed by means of a single question and rated according to a 5-point Likert scale (not at all, a little bit, a moderate amount, a great deal, and an extreme amount) and completeness of stool evacuation sensation evaluated by means of a single closed (yes/no) question in the patient's diary; (d) the Patient Assessment of ConstipationQuality of Life Questionnaire (PAC-QOL), a 28-item self-report questionnaire designed to evaluate the burden of constipation on patients' everyday HRQoL, covering the specific constipation-related domains of worries and concerns, physical discomfort, psychosocial discomfort, and satisfaction; each symptom's severity is referred to the 2 previous weeks and scored on a 5-point Likert scale, ranging from 0 (not at all) to 4 (extremely); and (e) the Global Patient Impression for Improvement Questionnaire (PGI-I), consisting of one question for the overall self-assessment of constipation improvement using a 7-point Likert scale (from 1-very much better, to 7-very much worse).

The objective of this study was to assess the safety and efficacy of naloxegol in a realworld setting in cancer patients. The primary safety endpoint was the incidence of adverse 
events leading to study discontinuation. The primary efficacy endpoint was the response rate during the 4 weeks of treatment. Response rate was defined as the proportion of participants reporting $\geq 3 \mathrm{BM}$ (without the use of rescue laxative treatment in the previous $24 \mathrm{~h}$ ) per week and an increase of $\geq 1 \mathrm{BM}$ over baseline.

Additionally, the following secondary endpoints were evaluated: (a) proportion of patients who had a change in their BFI score of $\geq 12$ points at the end of the study treatment (4 weeks) - this constitutes a clinically important improvement in OIC; (b) proportion of patients who had a BFI score of $<30$ at the end of the study-this constitutes well-controlled OIC; (c) time to the first post-dose BM; (d) change in stool consistency, according to the BSS [28]; (e) change in the Patient Assessment of Constipation-Quality of Life Questionnaire (PAC-QOL) and the four subscales (physical discomfort, psychosocial discomfort, worries and concerns, and satisfaction) [29]; (f) incidence of overall adverse events, including serious adverse events (SAEs); (g) analgesic treatment interruptions and dose adjustments; (h) naloxegol treatment interruptions and dose adjustments; and (i) patient satisfaction, assessed via the Patient Global Impression for Improvement Questionnaire (PGI-I).

\subsection{Statistical Analysis}

The study sample size was calculated based on the primary safety objective (the incidence of adverse events leading to study discontinuation). Previous studies showed that the incidence of adverse events leading to study discontinuation was about $10 \%$ after a follow-up of 12 weeks [19]. Therefore, an overall sample size of 315 patients would be necessary to detect $2 \%$ of discontinuations due to adverse events at 4 weeks, with a $95 \%$ confidence interval and a precision of $\pm 1.5 \%$.

Exploratory and descriptive methods were used to describe every study variable. All descriptive variables were tabulated. Continuous variables were described by the mean, median, standard deviation, minimum, maximum, and quartiles (range), and categorical variables were presented as distributions of frequencies and percentages. Comparisons of categorical variables were made using the Chi-square or Fisher's exact test, and for the comparison of quantitative variables, the paired-sample T-test was used. Besides this, associations between different study variables were analyzed using either the T-test for equality of means or the ANOVA test.

For the safety analysis, the population for the analyses comprised all patients who met all selection criteria and had received at least 1 dose of the study drug, while for the efficacy analysis, the population comprised all patients who met all selection criteria, had received at least 1 dose of the study drug, and had at least one post-baseline efficacy assessment. Specifically, two efficacy analyses were performed, based on the days of diary completion: a first one for those subjects who had completed both 4 weeks of treatment and 28 days of the diary (Efficacy Population 1), and a second one for those who had completed at least 21 days of the diary and were not study discontinuations (Efficacy Population 2).

All the data were analyzed using IBM SPSS Statistics version 22.0.

\section{Results}

From August 2018 to January 2020, 183 patients were screened, and 170 recruited by 26 centers from 10 European countries received at least one dose of naloxegol and were included in the safety population analysis. Participant centers included pain units, oncology departments, and palliative care units. Of the 170 patients, 143 patients who had at least one post-baseline efficacy assessment were included in the efficacy analysis; 76 patients were included in Efficacy Population 1 and 98 in Efficacy Population 2 (Figure 1). Overall, there were 56 (32.9\%) study discontinuations (Table S1). 


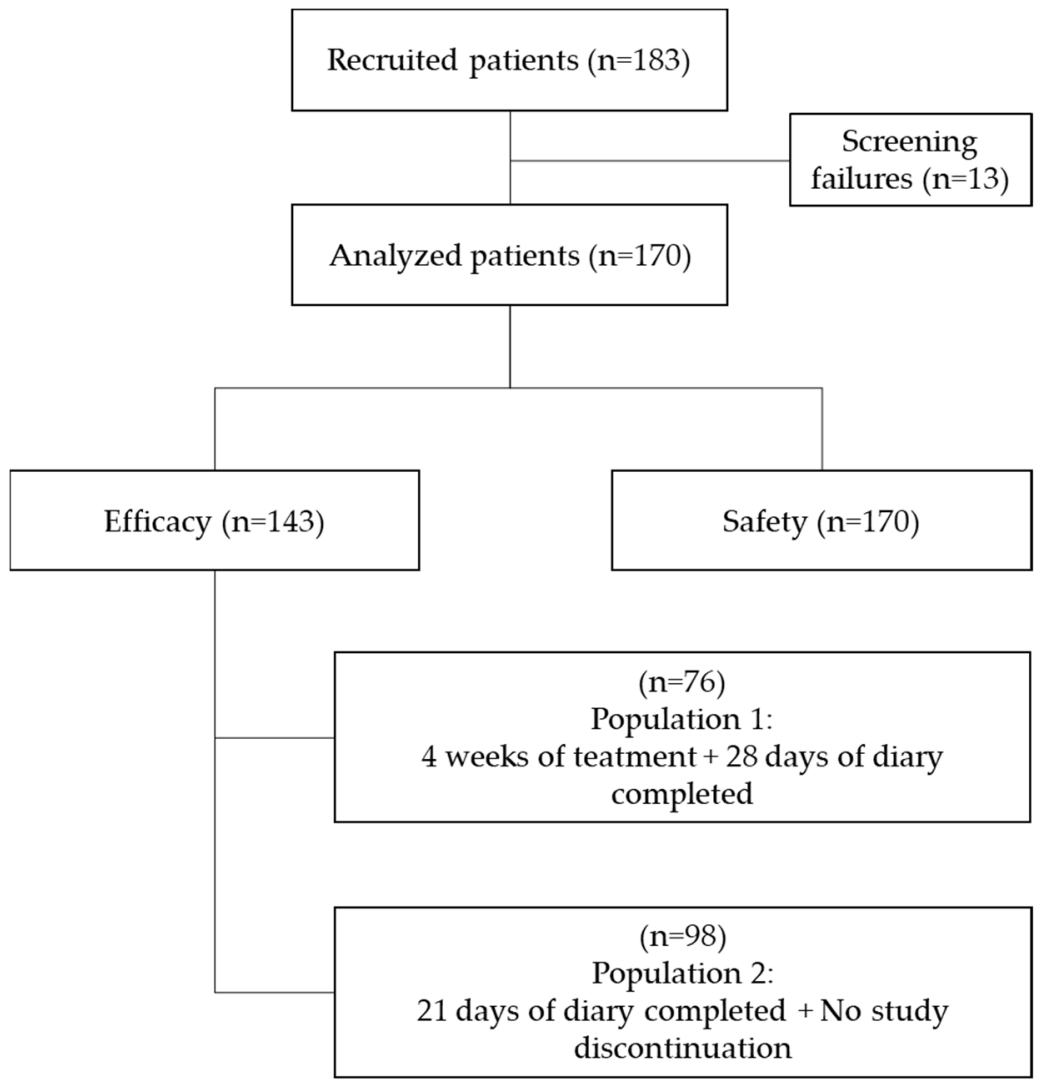

Figure 1. Disposition of subjects.

The baseline clinical and demographic characteristics of the patients included in the efficacy population are shown in Table 1 . The patients had a median (interquartile range $(\mathrm{IQR})$ ) age of 66 years (58-72) and were almost evenly distributed regarding gender. The most frequent primary tumor locations were lung $(n=35,24.5 \%)$ and breast $(n=32,22.4 \%)$, and $99(69.2 \%)$ patients presented metastasis. The most frequent opioids were fentanyl ( $n=38,26,6 \%)$, oxycodone $(n=36,25,2 \%)$, and morphine $(n=16,16.8 \%)$. Baseline opioid therapy was modified at week 2 in 31 of 140 patients (22.1\%), with dose increase, dose reduction, and opioid treatment change, in 20, 8, and 3 subjects, respectively; likewise, it was subsequently modified at week 4 in 22 of $118(18.6 \%)$ patients, with dose increase, dose reduction, and opioid treatment change, in 14 , 4 , and 4 subjects, respectively (Table S2).

Table 1. Baseline clinical and demographic characteristics.

\begin{tabular}{ccc}
\hline Baseline Characteristics & $\begin{array}{c}\text { Number Of } \\
\text { Analyzed Patients }\end{array}$ & Value \\
\hline Age, years, median (IQR) & 143 & $66.0(58.0 ; 72.0)$ \\
\hline Gender, female, N (\%) & 143 & $78(54.5)$ \\
\hline Race, Caucasian, N (\%) & 143 & $116(81.1)$ \\
\hline Height, cm, median (IQR) & 143 & $165.5(160.0 ; 171.0)$ \\
\hline Weight, kg, median (IQR) & 143 & $66.0(58.0 ; 78.0)$ \\
\hline Primary tumor location, N (\%) & 143 & \\
\hline Lung & 143 & $35(24.5)$ \\
\hline
\end{tabular}


Table 1. Cont.

\begin{tabular}{|c|c|c|}
\hline Baseline Characteristics & $\begin{array}{c}\text { Number Of } \\
\text { Analyzed Patients }\end{array}$ & Value \\
\hline Breast & 143 & $32(22.4)$ \\
\hline Prostate & 143 & $12(8.4)$ \\
\hline Pancreas & 143 & $10(7.0)$ \\
\hline Bladder & 143 & $6(4.2)$ \\
\hline Head and neck & 143 & $5(3.5)$ \\
\hline Uterus & 143 & $4(2.8)$ \\
\hline Bone & 143 & $3(2.1)$ \\
\hline Kidney & 143 & $3(2.1)$ \\
\hline Other & 143 & $33(23.0)$ \\
\hline Presence of metastasis, yes, N (\%) & 143 & $99(69.2)$ \\
\hline Current chemotherapy treatment, yes, N (\%) & 143 & $66(46.2)$ \\
\hline Carboplatin + Gemcitabine & 66 & $5(7.6)$ \\
\hline Nab-Paclitaxel + Gemcitabine & 66 & $4(6.1)$ \\
\hline Carboplatin + Paclitaxel & 66 & $3(4.5)$ \\
\hline Docetaxel & 66 & $3(4.5)$ \\
\hline Carboplatin + Etoposide & 66 & $2(3.0)$ \\
\hline \multicolumn{3}{|l|}{ Opioid treatment at Visit 1, N (\%) } \\
\hline Fentanyl & 143 & $38(26.6)$ \\
\hline Oxycodone & 143 & $36(25.2)$ \\
\hline Morphine & 143 & $16(11.2)$ \\
\hline Codeine * & 143 & $11(7.7)$ \\
\hline Oxycodone/Naloxone & 143 & $10(7.0)$ \\
\hline Hydromorphone & 143 & $8(5.6)$ \\
\hline Methadone & 143 & $7(4.9)$ \\
\hline Tramadol & 143 & $6(4.2)$ \\
\hline Fentanyl + Morphine & 143 & $2(1.4)$ \\
\hline Tramadol/paracetamol & 143 & $2(1.4)$ \\
\hline Tapentadol & 143 & $1(0.7)$ \\
\hline Other & 143 & $6(4.2)$ \\
\hline Previous laxative treatment at Visit 1, yes, N (\%) & 143 & $104(72.7)$ \\
\hline \multicolumn{3}{|l|}{ Previous laxative treatments at Visit $1, \mathrm{~N}(\%)^{* *}$} \\
\hline Osmotic & 104 & $83(79.8)$ \\
\hline Stimulant & 104 & $32(30.8)$ \\
\hline Stool softeners & 104 & $12(11.5)$ \\
\hline Bulking agents & 104 & $4(3.8)$ \\
\hline Linaclotide & 104 & $1(1.0)$ \\
\hline Other & 104 & $19(18.3)$ \\
\hline
\end{tabular}

IQR: Interquartile range. * It includes combinations of codeine plus paracetamol with or without caffeine. ** Percentages have been calculated over the total number of patients with previous laxative treatment $(n=104)$.

Patients could receive more than one laxative treatment. 
At baseline, 104 of 143 (72.7\%) subjects were receiving conventional laxatives, and the corresponding proportions at Visits 2 and 3 were 105/140 (75\%) and 43/118 (36.4\%), respectively. Osmotic and stimulant laxatives were the most frequently used at every study visit (data not shown). Furthermore, the most frequently used baseline laxatives were: osmotic laxatives $(79.8 \%)$, stimulant laxatives $(30.8 \%)$, stool softeners $(11.58 \%)$, and other (18.3\%, enema being the most common, with $9.6 \%)$.

Naloxegol $25 \mathrm{mg}$ daily was the initial treatment dose in 139 of 170 (81.8\%) patients, $30(17.6 \%)$ patients initiated treatment with naloxegol 12,5 mg daily, and 1 patient with naloxegol $50 \mathrm{mg}$ daily. At Visit 2, 14/140 (10\%) patients required a treatment interruption, and $4 / 140(2.8 \%)$ required a dose increase; the corresponding figures at Visit 3 were 11/118 $(7.8 \%)$ treatment interruptions and 6/118 (4.3\%) dose adjustments (the dose was increased in 5 patients, while the sixth patient had both an increased and a reduced dose adjustment).

\section{Safety}

Overall, 89 of 170 study subjects (52.4\%) reported at least one adverse event, and $38(22.4 \%)$ were recorded as serious adverse events. The most frequent (i.e., adverse events with an incidence rate of $\geq 5 \%$ ) are presented in Table S3. There were two cases of withdrawal syndrome that were categorized as grade 1.

Adverse events considered to be related to naloxegol were reported in 23 patients (13.5\%). Treatment-related adverse events were mainly gastrointestinal events, the most frequently reported being abdominal pain $(n=14,8.2 \%)$ and diarrhea $(n=5,2.9 \%)$. Except for a case of intestinal perforation that was categorized as grade 5 , the remaining evaluable adverse events were graded 1 to 3 (Table 2 ). There were two (1.2\%) treatment-related adverse events that were considered serious adverse reactions: a case of grade 5 intestinal perforation and a case of grade 2 diarrhea. The intestinal perforation occurred in a 68 years-old male, diagnosed with advanced pancreatic cancer, and with a medical history of gastric bypass surgery. Shortly after initiating treatment with $25 \mathrm{mg}$ of naloxegol, the patient experienced rapid deterioration of the general state, showed signs of peritonitis, sepsis and multiorgan failure, and finally died. Autopsy was not performed, and the event was categorized by the investigator as probable intestinal perforation.

Table 2. Adverse reactions to naloxegol (according to CTCAE v4.03).

\begin{tabular}{ccccccccc}
\hline \multirow{2}{*}{ Adverse Reaction } & \multicolumn{2}{c}{ Grade 1-3 } & \multicolumn{2}{c}{ Grade 4-5 } & \multicolumn{2}{c}{ Grade NA } & \multicolumn{2}{c}{ Total } \\
\cline { 2 - 9 } & $\mathbf{N}$ & $\mathbf{\%}$ & $\mathbf{N}$ & $\mathbf{\%}$ & $\mathbf{N}$ & $\mathbf{\%}$ & $\mathbf{N}$ & $\mathbf{\%}$ \\
\hline Abdominal pain & 10 & 5.9 & 0 & 0.0 & 4 & 2.4 & 14 & 8.3 \\
\hline Diarrhea & 4 & 2.4 & 0 & 0.0 & 1 & 0.6 & 5 & 2.9 \\
\hline Fatigue & 1 & 0.6 & 0 & 0.0 & 0 & 0.0 & 1 & 0.6 \\
\hline Flatulence & 2 & 1.2 & 0 & 0.0 & 0 & 0.0 & 2 & 1.2 \\
\hline Gastrointestinal pain & 1 & 0.6 & 0 & 0.0 & 0 & 0.0 & 1 & 0.6 \\
\hline Intestinal perforation & 0 & 0.0 & 1 & 0.6 & 0 & 0.0 & 1 & 0.6 \\
\hline Nausea & 2 & 1.2 & 0 & 0.0 & 0 & 0.0 & 2 & 1.2 \\
\hline Pollakiuria & 1 & 0.6 & 0 & 0.0 & 0 & 0.0 & 1 & 0.6 \\
\hline Vertigo & 1 & 0.6 & 0 & 0.0 & 0 & 0.0 & 1 & 0.6 \\
\hline Withdrawal syndrome & 1 & 0.6 & 0 & 0.0 & 0 & 0.0 & 1 & 0.6 \\
\hline
\end{tabular}

NA, not available; CTCAE: National Cancer Institute Common Terminology Criteria for Adverse Events. Grade 1: Mild; asymptomatic or mild symptoms; Grade 2: Moderate; minimal, local, or noninvasive; intervention indicated; Grade 3: Severe or medically significant but not immediately life-threatening; Grade 4: Life-threatening consequences; urgent intervention indicated; Grade 5: Death related to AE.

Out of 170 patients, $20(11.8 \%, 95 \%$ CI 6.9-16.6) discontinued the study due to adverse events, and, of them, 12 (7.1\%, 95\%CI 3.2-10.9\%) were study discontinuations due to naloxegol-related adverse events. These were mainly gastrointestinal side effects, including 
eight cases of abdominal pain, two cases of diarrhea, and one due to intestinal perforation; the remaining patient discontinued the study due to fatigue.

\section{Efficacy}

\subsection{Bowel Movements}

A total of fifty-five patients $(72.4 \%, 95 \%$ CI 62.3\%-82.4\%) from Population $1(\mathrm{~N}=76)$ met the primary efficacy endpoint after the 4-week study follow-up period, and they were regarded as responders to naloxegol treatment. After the 4-week study period, the average number of weekly BMs in Population 1 was 6.9 (95\% CI, [6.1-7.7]), split into 8.1 (95\%, CI (7.3-8.9)) and 3.7 (95\% CI, (3.2-4.2)) for the responders and non-responders, respectively $(p<0.001)$ (Figure 2). Likewise, 74 patients $(75.5 \%, 95 \%$ CI 67.0\%-84.0\%) from Population $2(n=98)$ responded to naloxegol treatment. The results regarding the number of weekly BMs in Population 2 almost overlapped those of Population 1 (Figure 2).

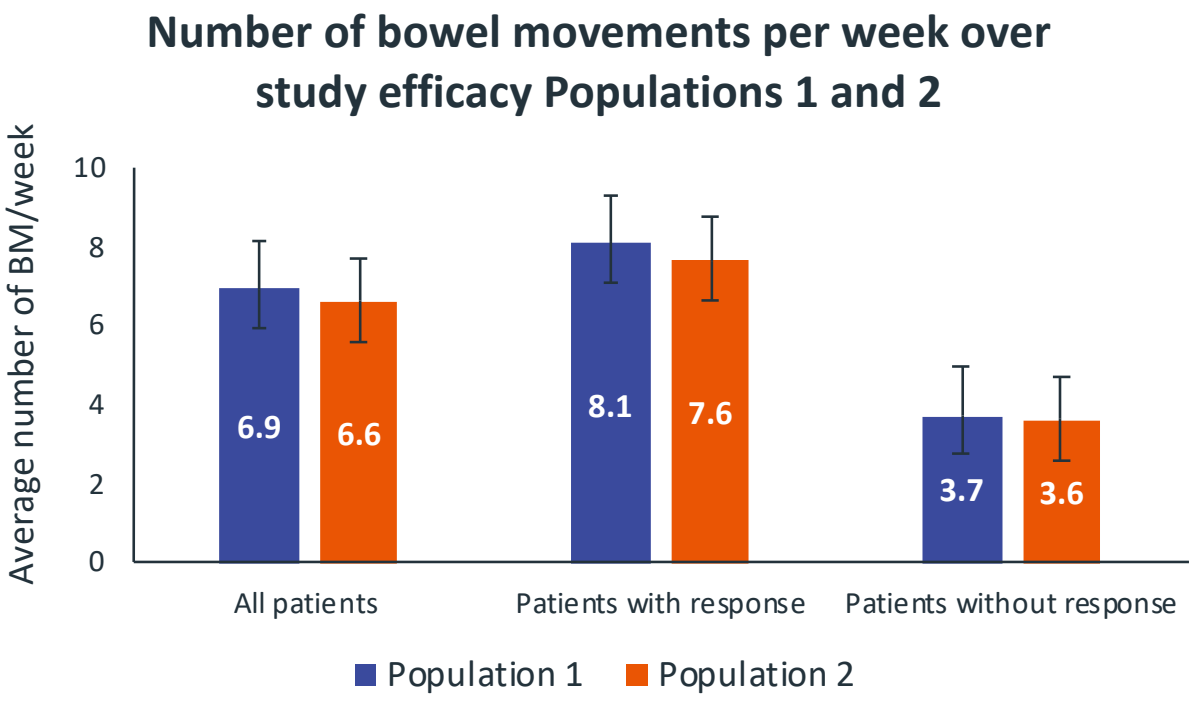

Figure 2. Average number of bowel movements per week over study efficacy Populations 1 and 2.

BM, bowel movement.

Vertical bars represent $95 \%$ confidence intervals.

Population 1: Subjects who had completed both 4 weeks of treatment and 28 days of the diary.

Population 2: Subjects who had completed at least 21 days of the diary.

The response to naloxegol was mainly observed within the first week of treatment. The mean number (standard deviation, SD) of BMs per week increased from $<3$ at baseline to 7.6 (5.0) and 7.3 (4.8) at Week 1 in Populations 1 and 2, respectively. There were no statistically significant differences in the average number of weekly BMs between Weeks 1 and 4 in both populations. The mean (SD) time to the first post-dose bowel movement was 1.9 (1.7) days.

\subsection{Other Efficacy Outcomes}

Of 117 evaluable patients, 75 (64.1\%, 95\% CI 55.4-72.8\%) had a BFI score change of $\geq 12$ points at the end of the study. Likewise, 43 patients (36.8\%, 95\% CI $28.1-45.5 \%)$ had a BFI score of $<30$ at the study end.

Stool consistency as assessed via the BSS increased by a mean of 0.8 (1.8) points by Week 2 and 0.9 (1.8) points by Week 4. Pairwise comparison showed a significant improvement in the BSS score from baseline to Week $4(p<0.001)$; (detailed information on the BSS results is presented in Table S4).

The number (percentage) of patients reporting a great deal or an extreme amount of straining during bowel movement decreased from 67 out of 135 evaluable patients (49.6\%) 
at baseline to 22 out of 110 evaluable patients (20.0\%) at Week 4 (Figure 3). A significant improvement in self-reported straining perception was observed from baseline to Week 2 $(p=<0.001)$ and to Week $4(p<0.001)$ according to pairwise comparisons.

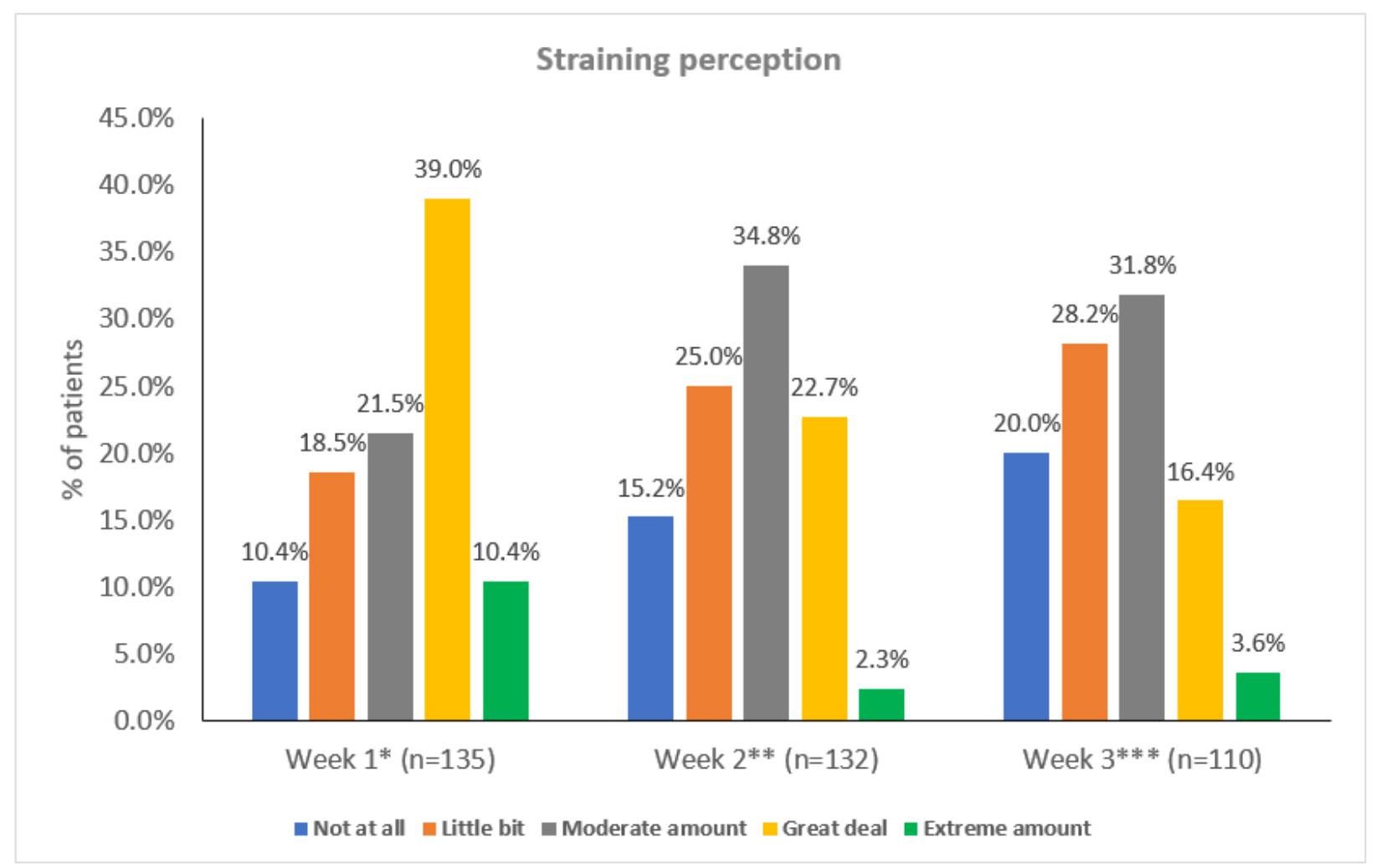

Figure 3. Straining perception during the study. ${ }^{*} 8$ missing data. ${ }^{* *} 11$ missing data. ${ }^{* * *} 9$ missing data. Note: 24 patients did not complete Visit 3.

The number and proportion of patients with sensation of incomplete evacuation decreased from 109 of 137 evaluable subjects (79.6\%) at baseline to 80 of 132 subjects $(60.6 \%)$ at Week 2 and to 63 of 113 subjects (55.6\%) at the end of Week 4 of treatment. Based on the pairwise comparisons, the difference in the proportions of patients with sensation of incomplete evacuation between baseline and Week 2 was statistically significant $(p=0.007)$, while that between baseline and Week 4 did not reach statistical significance $(p=0.089)$ (data not shown).

PAC-QOL total score and all its subscales improved from baseline to 4 weeks of follow up. The proportion of patients with clinically relevant improvement in the total PAC-QOL score was $37.1 \%(n=124)$. The mean for baseline, Week 2 and Week 4 for the PAC-QOL total score and subscale scores are displayed in Figure 4. All the changes in the PAC-QOL scores reached statistical significance in the pairwise comparisons.

A total of one hundred and eighteen patients $(75.0 \%, 95 \% \mathrm{CI} 67.2-82.8 \%)$ reported that their constipation had improved, according to the PGI-I questionnaire, after 4 weeks of treatment. A total of fifty-one (43.2\%) patients were "much better" or "very much better".

Notably, a direct association between the responses to the HrQoL PAC-QOL (total score) and PGI-I questionnaires was observed $(p<0.001)$. 
Evolution of PAC-QOL score and subscales

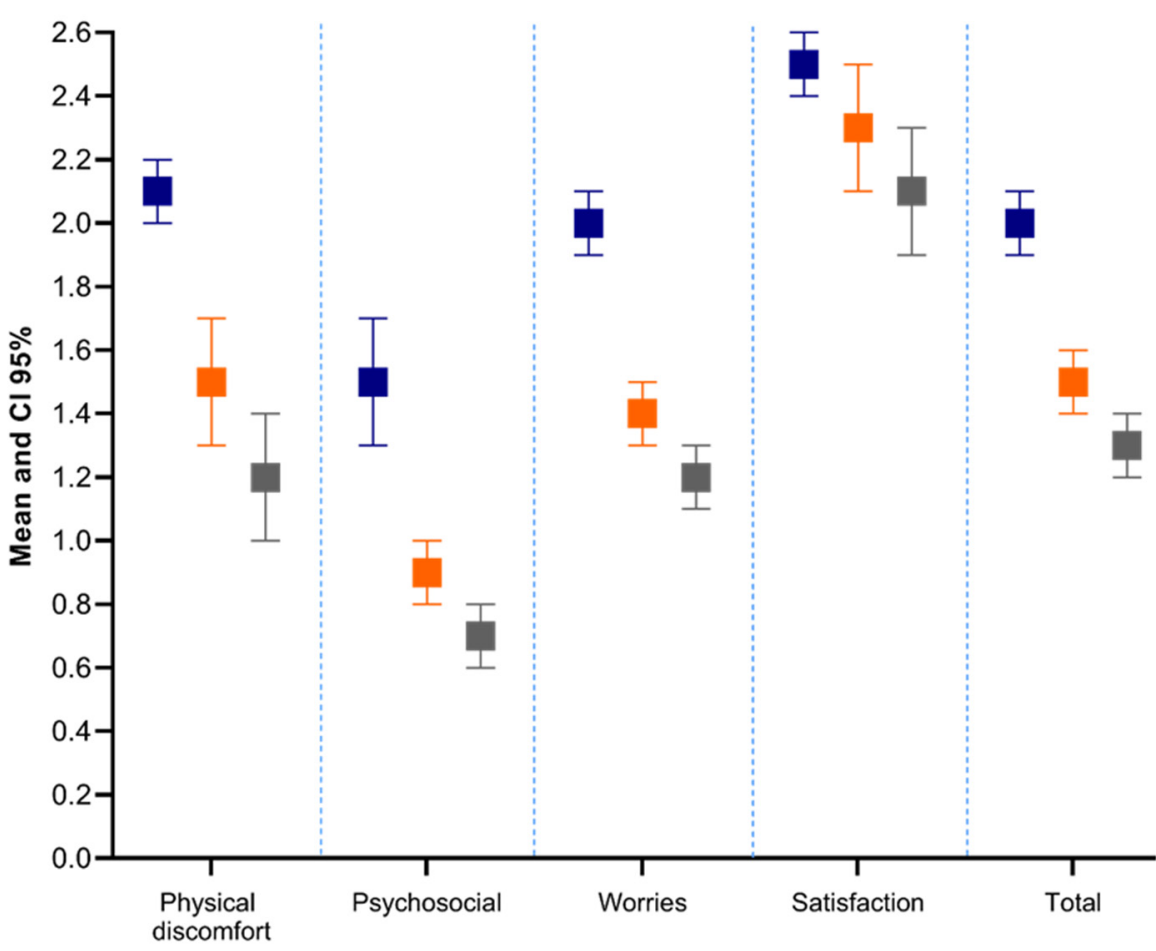

Figure 4. PAC-QOL changes throughout the study.

\section{Discussion}

We conducted a multinational (European), multicenter, prospective, real-world observational study aimed to evaluate the efficacy and safety of naloxegol for the treatment of OIC in cancer patients after a 4-week follow-up period. The results from our study support the generally good tolerability and safety profile of naloxegol and its efficacy in patients with cancer-related pain and OIC treated under routine clinical practice conditions. Furthermore, based on these data the efficacy of naloxegol appears to be independent of the type of opioid and relieves OIC quickly.

There was a small proportion of subjects who reported adverse reactions due to naloxegol treatment $(13.5 \%)$, and only $7.1 \%$ of the whole safety population discontinued early from the study as a consequence of naloxegol-related adverse events. This proportion is slightly lower than the $10 \%$ reported in phase III, 12-week clinical trials [19], and is within the range reported in similar real-world prospective observational studies: $4.8 \%$ after 12 months but all of them within the first 4 weeks [22], and $6.8 \%$ after 4 weeks [23], respectively. It is worth mentioning that most of these adverse events frequently occurred within the first two weeks of treatment with naloxegol [20,22]; therefore, it is also possible that most adverse reactions leading to drug discontinuation occur early during treatment. The tolerability profile was consistent with those reported in both clinical trials $[19,20]$ and observational studies [22,23], the most commonly reported naloxegol-emergent adverse events in our study being of gastrointestinal nature and of mild to moderate severity.

Regarding the primary efficacy endpoint, a high proportion of study patients $(72.4 \%)$ responded to naloxegol treatment during the 4-week study follow-up period. This result is also consistent with the reported percentages from two other similar observational studies with naloxegol, in which the response rate was assessed by the number of BMs over the previous weeks $[22,23]$. 
In a 12-month follow-up observational study (Kyonal Study), Cobo et al. showed the sustained efficacy and safety of naloxegol improving OIC, quality of life as measured with the PAC-QOL, and symptoms of cancer patients [22]. A more recent 4-week followup study (MovE Study), conducted in France in patients with cancer and OIC, receiving naloxegol treatment also showed high response rates to naloxegol, with an improvement of constipation that was associated with an improvement of quality of life [23]. After 4 weeks of treatment with naloxegol, Cobo et al. in the Kyonal Study reported a response rate of $74.6 \%$ [22], and Lemaire et al. in the MovE Study found a response rate of $73.4 \%$ [23]. These results are better than those reported in the phase III trials, where investigators observed response rates of $48.7 \%$ and $46.8 \%$ at Week 12 in the KODIAC-04 and KODIAC-05 studies, respectively [19]. However, our definition of response (i.e., $\geq 3$ SBM per week, without the use of rescue laxative treatment in the previous $24 \mathrm{~h}$, and an increase of $\geq 1$ SBM over baseline) differs from the definition used in clinical trials (i.e., $\geq 3$ SBM per week and an increase from baseline of $\geq 1$ SBM for $\geq 9$ of 12 weeks and for $\geq 3$ of the final 4 weeks). Therefore, the response rates with naloxegol from observational studies and clinical trials are hardly comparable.

In our study, we consistently found significant improvements in most of the secondary efficacy endpoints, despite the short study duration. Statistically (and, more importantly, clinically) significant improvements at the end of the study were observed in the selfperception problems associated with constipation (as measured via the BFI questionnaire), stool consistency (as evaluated through the BSS), and self-reported straining perception. Furthermore, the quality of life of the study subjects had also improved by the end of the study, as reflected by the changes in the PAC-QOL total score and all its subscale scores. This was further corroborated by the significant improvement in the self-perception of efficacy according to the PGI-I questionnaire and by the finding of a significant association between the PAC-QOL total score and PGI-I.

The NACASY study is the first multinational European study evaluating naloxegol for the treatment of OIC in cancer patients in a real-world setting. The limitations of this study are those stemming from its noninterventional design and the absence of a control group, so our findings should be considered supportive of those reported in randomized controlled trials. Missing data in observational studies are an issue and could be responsible for an overestimation of treatment effects compared to the intention-to-treat analysis of the randomized controlled trials. We did not achieve the sample size foreseen in the protocol, increasing the imprecision of our results.

\section{Conclusions}

The findings from the NACASY study support and provide new evidence about the beneficial effect of naloxegol in terms of improvement of constipation and quality of life in patients with cancer-related pain and opioid-induced constipation, with a tolerability and safety profile consistent with that reported by Cobo et al. and Lemaire et al. in the Kyonal and MovE observational studies in patients with cancer, respectively, as well as those reported in phase III clinical trials in patients with noncancer pain. Currently, and despite its high prevalence, OIC remains under-recognized and undertreated, thus impacting on wellbeing and quality of life of cancer patients [30]. In this study, the authors highlight the importance of using precise and practical tools for an efficient OIC diagnosis and management. Taken together, the potential occurrence of OIC should be considered from the start of opioid therapy in cancer patients, allowing patients to receive the right treatment at the right time, thus improving treatment outcomes in this population.

Supplementary Materials: The following supporting information can be downloaded at: https:/ /www. mdpi.com/article/10.3390/cancers14051128/s1, Table S1: Study discontinuations; Table S2: Opioid treatment modifications during the study; Table S3: Most common ( $>5 \%$ ) adverse events; Table S4: Changes in stool consistency according to the BSS. 
Author Contributions: Each author has participated sufficiently to take public responsibility for the manuscript content. Conceptualization, A.D. and J.T.; methodology, A.D. and J.T.; investigation, S.C., D.D., S.E.F., J.L., V.M., K.M. and J.S.; formal analysis, A.D. and J.T.; writing-original draft, A.D. and J.T.; writing-review and editing, S.C., D.D., S.E.F., J.L., V.M., K.M. and J.S. All authors have read and agreed to the published version of the manuscript.

Funding: This project was funded by Kyowa Kirin International. Role of the funding source: The funder of the study and its employees and assignees were involved in study design, data collection, data analysis, data interpretation, and writing of all related reports and publications.

Institutional Review Board Statement: The study was reviewed and approved by the Ethics Committees from Hospital Universitari Vall d'Hebron, Barcelona, Spain (Code NACASY [KKF-NAL-201801], from the Hospital Universitari Germans Trias i Pujol, Badalona, Spain (Code CEI PI-18-085), from Hospital Clinico San Carlos, Madrid, Spain (Code 18/154-O_SP), Comitatto Etico per la Sperimentazione Clinica delle Province di Verona e Rovigo (Code 82430), Comitato Indipendente di Etica Medica, Brindisi, Italy (Code 178/18), Comitato Etico Reagionale delle Marche, Torrette Di Ancona, Italy, Raad van Bestuur van Rijnstate Arnhem, Nederlands (2018-1225), Comité de Protection des Personnes Ile-de-France X, Aulnay-sous-bois, France (Code 2018-A00845-50) and Dulwich Research Ethics Committee, London, United Kingdom (Code 18/LO/2056).

Informed Consent Statement: Written informed consent was obtained from all subjects before enrollment in the study.

Data Availability Statement: Data will be available on request due to restrictions e.g., privacy or ethical.

Acknowledgments: Kyowa Kirin International and the authors thank the patients and their families, study sites, and investigators who participated in this clinical study. The further members of the NACASY Study Group (in alphabetical order): Sonja Aho, Tampere University Hospital, Tampere, Finland; Michael Bennett, Gemma's Hospice, Leeds, United Kingdom; Klaus Bitsch Jakobsen, Aalborg Universitets Hospital Farsø, Farsø, Denmark; Cristina Caramés, Hospital Universitario Fundación Jiménez Díaz, Madrid, Spain; Saverio Cinieri, Ospedale A. Perrino, Brindisi, Italy; Andrew Davies, Royal Surrey Hospital, Guildford, United Kingdom; Denis Dupoiron, Institut de Cancérologie de l'Oues (Paul Papin Angers) Angers, France and Institut de Cancérologie de l'Ouest (Centre René Gauducheau), Sant Herblain, France; Gabriella Farina, ASST Fatebenefratelli Hospital, Milan, Italy; Cristina Farriols, Hospital del Mar, Barcelona, Spain; Stefania Gori, Hospital Negrar, Negrar, Italy; Hortense Thelu, Institut Gustave-Roussy, Villejuif, France; Mathieu Laramas, Centre Hospitalier Universitaire Grenoble, Grenoble, France; Johan Leclerc, Hopital Nord, Amiens, France; Bertrand Mennecier, Centre Hospitalier Universitaire de Strasbourg, Strasburg, France; Sofía España, Institut Català d'Oncologia Badalona (ICO), Barcelona, Spain; Vincenzo Montesarchio, AORN Dei Colli (Ospedale Monaldi), Naples, Italy; Reinhard Musch, Krebsheilkunde Lichtenberg, Berlin, Germany; Kyriaki Mystakidou, Palliative Care Unit "Jenny Karezi", Athens, Greece; Pedro Pérez Segura, Hospital Clínico San Carlos, Madrid, Spain; Mikael Segerlantz, Skåne University Hospital, Lund, Sweden; Judith Serna, Hospital Universitari Vall d'Hebron, Barcelona, Spain; Michael Sohm, Tagesklinik Landshut, Landshut, Germany; Marieke Van den Beuken, Maastricht, Netherlands; Maurice Van der Vost, Rijnstate, Arnhem, Netherlands; Ruth Vera, Complejo Hospitalario de Navarra, Pamplona, Spain. We also thank Juan Luis Sanz and Susana Vara (APICES, Madrid; Spain) for their support with the study design, setup, coordination and project management, monitoring, and statistical analysis, and Fernando Rico-Villademoros (APICES, Madrid, Spain) for writing a draft of this manuscript and his editorial assistance; their participation has been funded by Kyowa Kirin International.

Conflicts of Interest: Andrew Davies has received research funding from Kyowa Kirin to undertake investigator initiated studies into opioid-induced constipation; he has also receive honoraria for speaking at symposia, and to take part in advisory boards. Saverio Cinieri has no conflict of interest to declare; Denis Dupoiron received consultancy fees from Medtronic, Grunenthal and Esteve; Sofia España Fernandez has no conflict of interest to declare; Johan Lecrerc has no conflict of interest to declare; Vincenzo Montesarchio has no conflict of interest to declare; Kyriaki Mystakidou has no conflict of interest to declare; Judith Serna has no conflict of interest to declare; Jan Tack has given scientific advice to Adare, AlfaWassermann, Arena, Bayer, Christian Hansen, Clasado, Danone, Devintec, Falk, Grünenthal, Ironwood, Janssen, Kiowa Kirin, Menarini, Mylan, Neurogastrx, Neutec, Novartis, Nutricia, Ricordati Shionogi, Takeda, Truvion, Tsumura, Zealand, and Zeria pharmaceuticals, has 
received research support from Biohit, Shire, Sofar and Takeda, and has served on the Speaker bureau for Abbott, Allergan, AstraZeneca, Janssen, Kyowa Kirin, Menarini, Mylan, Novartis, Shire, Takeda, Wellspect, and Zeria.

\section{References}

1. Neufeld, N.J.; Elnahal, S.M.; Alvarez, R.H. Cancer pain: A review of epidemiology, clinical quality and value impact. Future Oncol. 2017, 13, 833-841. [CrossRef]

2. Fallon, M.; Giusti, R.; Aielli, F.; Hoskin, P.; Rolke, R.; Sharma, M.; Ripamonti, C.I. Management of cancer pain in adult patients: ESMO Clinical Practice Guidelines. Ann. Oncol. 2018, 29, iv166-iv191. [CrossRef]

3. Chwistek, M. Recent advances in understanding and managing cancer pain. F1000Research 2017, 6, 945. [CrossRef]

4. Chou, R.; Fanciullo, G.J.; Fine, P.G.; Adler, J.A.; Ballantyne, J.C.; Davies, P.; Donovan, M.I.; Fishbain, D.A.; Foley, K.M.; Fudin, J.; et al. Clinical guidelines for the use of chronic opioid therapy in chronic noncancer pain. J. Pain 2009, 10, 113-130. [CrossRef]

5. Mercadante, S. Opioid Analgesics Adverse Effects: The Other Side of the Coin. Curr. Pharm. Des. 2019, 25, 3197-3202. [CrossRef]

6. Farmer, A.D.; Drewes, A.M.; Chiarioni, G.; De Giorgio, R.; O’Brien, T.; Morlion, B.; Tack, J. Pathophysiology and management of opioid-induced constipation: European expert consensus statement. United Eur. Gastroenterol. J. 2019, 7, 7-20. [CrossRef]

7. Argoff, C.E. Opioid-induced Constipation: A Review of Health-related Quality of Life, Patient Burden, Practical Clinical Considerations, and the Impact of Peripherally Acting $\mu$-Opioid Receptor Antagonists. Clin. J. Pain 2020, 36, 716-722. [CrossRef]

8. Bell, T.J.; Panchal, S.J.; Miaskowski, C.; Bolge, S.C.; Milanova, T.; Williamson, R. The prevalence, severity, and impact of opioid-induced bowel dysfunction: Results of a US and European Patient Survey (PROBE 1). Pain Med. 2009, 10, 35-42. [CrossRef]

9. Kumar, L.; Barker, C.; Emmanuel, A. Opioid-Induced Constipation: Pathophysiology, Clinical Consequences, and Management. Gastroenterology Res. Pract. 2014, 2014, 141737. [CrossRef]

10. Larkin, P.J.; Cherny, N.I.; La Carpia, D.; Guglielmo, M.; Ostgathe, C.; Scotté, F.; Ripamonti, C.I. Diagnosis, assessment and management of constipation in advanced cancer: ESMO Clinical Practice Guidelines. Ann. Oncol. 2018, 29, iv111-iv125. [CrossRef]

11. Pergolizzi, J.V., Jr.; Christo, P.J.; LeQuang, J.A.; Magnusson, P. The Use of Peripheral $\mu$-Opioid Receptor Antagonists (PAMORA) in the Management of Opioid-Induced Constipation: An Update on Their Efficacy and Safety. Drug Des. Dev. Ther. 2020, 14, 1009-1025. [CrossRef] [PubMed]

12. Streicher, J.M.; Bilsky, E.J. Peripherally Acting $\mu$-Opioid Receptor Antagonists for the Treatment of Opioid-Related Side Effects: Mechanism of Action and Clinical Implications. J. Pharm. Pract. 2018, 31, 658-669. [CrossRef] [PubMed]

13. Emmanuel, A.; Johnson, M.; McSkimming, P.; Dickerson, S. Laxatives Do Not Improve Symptoms of Opioid-Induced Constipation: Results of a Patient Survey. Pain Med. 2017, 18, 1932-1940. [CrossRef] [PubMed]

14. Pappagallo, M. Incidence, prevalence, and management of opioid bowel dysfunction. Am. J. Surg. 2001, 182, 11s-18s. [CrossRef]

15. Camilleri, M.; Drossman, D.A.; Becker, G.; Webster, L.R.; Davies, A.N.; Mawe, G.M. Emerging treatments in neurogastroenterology: A multidisciplinary working group consensus statement on opioid-induced constipation. Neurogastroenterol. Motil. 2014, 26, 1386-1395. [CrossRef]

16. Fernández-Montes, A.; de Velasco, G.; Aguín, S.; Farriols, C.; Guirado-Risueño, M.; Jerviz-Guía, V.G.; Baeza-Nadal, M.V.; Chicas-Sett, R.; Fírvida, J.L.; García-Navalón, F.; et al. Insights into the Use of Peripherally Acting $\mu$-Opioid Receptor Antagonists (PAMORAs) in Oncologic Patients: From Scientific Evidence to Real Clinical Practice. Curr. Treat. Options Oncol. 2021, 22, 26. [CrossRef]

17. FDA Okays Naloxegol (Movantik) in Opioid-Induced Constipation. Available online: http://www.medscape.com/viewarticle/ 831780 (accessed on 24 September 2014).

18. European Medecine Agency. Moventig. Summary of Product Characteristics. Available online: https://www.ema.europa.eu/en/ documents/product-information/moventig-epar-productinformation_en.pdf. (accessed on 10 June 2020).

19. Chey, W.D.; Webster, L.; Sostek, M.; Lappalainen, J.; Barker, P.N.; Tack, J. Naloxegol for opioid-induced constipation in patients with noncancer pain. N. Engl. J. Med. 2014, 370, 2387-2396. [CrossRef]

20. Webster, L.; Chey, W.D.; Tack, J.; Lappalainen, J.; Diva, U.; Sostek, M. Randomised clinical trial: The long-term safety and tolerability of naloxegol in patients with pain and opioid-induced constipation. Aliment. Pharmacol. Ther. 2014, 40, 771-779. [CrossRef]

21. Webster, L.; Tummala, R.; Diva, U.; Lappalainen, J. A 12-week extension study to assess the safety and tolerability of naloxegol in patients with noncancer pain and opioid-induced constipation. J. Opioid Manag. 2016, 12, 405-419. [CrossRef]

22. Cobo Dols, M.; Beato Zambrano, C.; Cabezón-Gutiérrez, L.; Chicas-Sett, R.; Blancas López-Barajas, M.I.; García Navalón, F.J.; Fírvida Pérez, J.L.; Serrano Bermúdez, G.; Togores Torres, P.; Delgado Mingorance, I.; et al. One-year efficacy and safety of naloxegol on symptoms and quality of life related to opioid-induced constipation in patients with cancer: KYONAL study. BMJ Support Palliat. Care 2021. [CrossRef]

23. Lemaire, A.; Pointreau, Y.; Narciso, B.; Piloquet, F.X.; Braniste, V.; Sabaté, J.M. Effectiveness of naloxegol in patients with cancer pain suffering from opioid-induced constipation. Support Care Cancer 2021, 29, 7577-7586. [CrossRef] [PubMed] 
24. Association, W.M. World Medical Association Declaration of Helsinki: Ethical Principles for Medical Research Involving Human Subjects. JAMA 2013, 310, 2191-2194. [CrossRef]

25. International Ethical Guidelines for Epidemiological Studies. Available online: https://cioms.ch/wp-content/uploads/2017/01/ International_Ethical_Guidelines_LR.pdf. (accessed on 25 November 2021).

26. Tack, J.; Drossman, D.A. What's new in Rome IV? Neurogastroenterol. Motil. 2017, 29, e13053. [CrossRef] [PubMed]

27. Lacy, B.E.; Mearin, F.; Chang, L.; Chey, W.D.; Lembo, A.J.; Simren, M.; Spiller, R. Bowel Disorders. Gastroenterology 2016, 150, 1393-1407. [CrossRef]

28. Lewis, S.J.; Heaton, K.W. Stool form scale as a useful guide to intestinal transit time. Scand J. Gastroenterol. 1997, 32, 920-924. [CrossRef]

29. Marquis, P.; De La Loge, C.; Dubois, D.; McDermott, A.; Chassany, O. Development and validation of the Patient Assessment of Constipation Quality of Life questionnaire. Scand. J. Gastroenterol. 2005, 40, 540-551. [CrossRef]

30. Davies, A.; Leach, C.; Butler, C.; Gregory, A.; Henshaw, S.; Minton, O.; Shorthose, K.; Batsari, K.M. Opioid-induced constipation in patients with cancer: A 'real-world,' multicentre, observational study of diagnostic criteria and clinical features. Pain 2021, 162, 309-318. [CrossRef] 